\title{
MODERIZATION OF CORTICALLY SUPPORTED INDIVIDUAL IMPLANTS
}

\author{
Klaudia KULCSÁR ${ }^{1}$, János KÓNYA ${ }^{2}$ \\ ${ }^{1}$ Dent-Art-Technik Kft. Györ, Hungary \\ ${ }^{1}$ kulcsar.klaudia@dentarttechnik.hu \\ 2labor@dentarttechnik.hu
}

\begin{abstract}
Digital product processing, 3D and finite element analysis techniques, modern, tissue-friendly implants with adequate surface treatment and proper screw-fixing make it possible for a good idea to successfully treat patients who do not have a sufficient amount of bone tissue for the implantation of conventional cylindrical-shaped dental implants. The duration of the surgical procedure of a complete, toothless jawbone becomes much shorter. Complicated measurements and risky techniques that require substantial expertise become unnecessary. The implant can be placed precisely into position with a simple surgical procedure. Then, it can be fixed to the bone tissue with the help of pre-designed fixation points. After this, only the professional closing of the wound has to be carried out. We have designed and manufactured a titanium cortical implant that is novel both in the view of chemical composition and surgical procedure. The final connection shaping of the sleeve and abutment system of the personalized cortical-backup implants was designed with the help of finite element analysis methods after the healing process.
\end{abstract}

Keywords: Finite Element Analysis, Titanium, Subperiosteal Implant, Dental Implants, Patient-specific Implants

\section{Introduction}

The first subperiosteal implant was introduced by Dahl at the beginning of the 1940s. It was placed on the jawbone without any fixation [1]. During this procedure, the gum tissue of the patient was opened up so that the toothless jawbone was revealed. Then the geometry of the jawbone was imprinted with the use of a sterilized imprint material. After this, the wound was closed. A cast was created based on the geometry of the imprinted sample. The cast made it possible to design and manufacture a subperiosteal frame, which fitted to the bone tissue. It was made of nickel-cobalt-chromium alloy and was equipped with holes enabling screwfixation to the bone if needed. There were vertical nesting surfaces (connection points) going through the gum tissue at given parts of the framework to fix the denture. After the high-precision metal (Vitallium) framework was sterilized, the patient's gum tissue was opened up again. The framework was positioned onto the jawbone and fixed with screws if necessary. Finally, the gum tissue was closed back again [2].

The disadvantage of Dahl's procedure was that the framework was not integrated into the bone tissue, only the patient's connective tissue held it in position. 
Because of the complicated surgical procedure that had to be carried out multiple times, and the uncertain success rate, the procedure was abandoned over time. However, in a new context, the fixing of subperiosteal implants into the jawbone, instead of drilling several implants into the bone tissue, can be an innovative technique [3].

Generally speaking, axial implant loading and the axial implant-abutment connection is preferred in dental technology. These contribute to the longterm prosthetic success of dental implantation. The implant's improper position, cleanability and biomechanical position lead to "peri-implantitis" (a destructive inflammatory process affecting tissues nearby the implant) with a higher probability. This can lead to bone loss and the loss of the implant.

The idea behind our innovative approach is a personalized dental replacement procedure which avoids risk factors of "peri-implantitis" in the case of patients with extra hard or soft tissue. These patients used to be treated with timeconsuming, expensive, multiple-step treatments [4], [5].

Completely rethinking the process of subperiosteal implantation, we have changed the whole method: we use digital 3D-CBCT imaging to obtain bone geometry, we generate our design in a virtual environment and carry out the important statical analyses. The result is manufactured using titanium, which is osseointegration, so it can integrate into bone tissue. A surface finishing procedure is performed, and up-to-date technological possibilities are used for the structural analysis of the finished product.

Figure 1. shows the first subperiosteal implant we developed. After rethinking the subperiosteal implant several times and changing its manufacturing process, we named it cortically supported the individual implant.

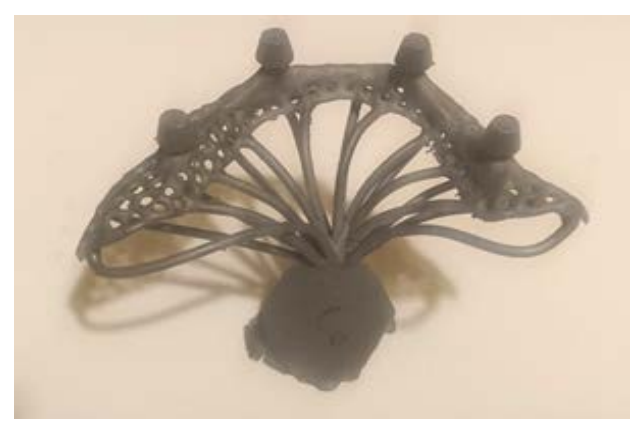

Figure 1. Subperiosteal implant

\section{Materials and methods in the implantation procedure of cortically supported individual implant}

\subsection{Criteria of use}

Cortically supported individual implant can be used in any tooth deficiency where implantation and bone replacement are needed. The titanium framework placed around the jawbone is optimal because it protects the neighbouring bone graft from the pressure of mucous membrane and from other mechanical stimuli. Furthermore, its pitted structure enables bone tissue to grow all around and integrate the implant, as the use of titanium results in osseointegration. A special surface treatment is also used to enhance this.

Among the criteria of the procedure is 3D-CBCT imaging - 3D-printing of jawbone from a polymeric material, precision denture and bite mould, and also polymeric jawbone - for the positioning of the polymeric jawbone, the conventional cast and the permanent denture.

\subsection{Work process in dental technician laboratory}

\subsubsection{The preparation process in the dental technical laboratory}

The geometry and position of the patient's teeth are designed manually using 
the Wax-up cast, which is created based on the mould. After this, a proposal is made for optimal virtual design, dimensions and quantity of the support material needed under the patient's teeth.

\subsection{2. $3 D$ design}

All the required geometrical data for computer-aided design is derived from Digital Volume Tomography (also called CBCT - Cone Beam CT) images of the patient. In this case, data can be derived in DICOM format from CB-CT apparatus made for dental applications. We convert DICOM format to STL. This 3D data format can be processed by numerous types of computer software, which helps design the subperiosteal implant that fits perfectly to the jawbone. The result of the computeraided design is the geometry which fulfils both the medical and prosthetic requirements completely. We scan the wax or self-curing resin model, which simulates the replacement itself, then convert the digital data for 3D-printing. A 3D-printer is utilized to manufacture the cortically supported individual implant that we designed. The product serves as a mould for titanium casting, with its special-purpose photopolymeric material that can be burned out without slag.

\subsubsection{Manufacturing the cortically supported individual implant}

The 3D-printed polymeric framework geometry is cast with a vacuum pressure casting machine using titanium. The finished titanium cast is tested by CT X-ray analysis to eliminate inclusions and dislocations that can affect planned mechanical strength. This test allows further processing only of those casts which are suitable for further use due to their homogeneity. After the removal of casting gates, the workpiece is given a surface treatment. This is a surface melting process using a scanning laser beam. After this, the homogeneous surface is subjected to a dorsal (area of bone side) micro-ceramic surface treatment, which enhances osseointegration.

\subsection{Surgical procedure}

The metal and polymeric parts are sterilized in the operation room before surgery. Using local anaesthesia, the jawbone is revealed with a section on the ridge. This section is just enough big for the titanium framework to fit in.

The surface of the bone is perforated in several places to enable vascularization through the cortical part of the bone to the bone graft. This helps transform bone graft to living bone tissue. The framework is placed onto the perforated tissue. Its proper position is checked and bone tissue can be adjusted for perfect mating. Afterwards, it is filled with a mixture of bone graft and the patient's own bone tissue. After proper positioning, it is fixed by screws at certain locations. The mucosal flap is elongated by cleaving the periosteum to a certain extent and closed tension-freely over the titanium frame. We wait for osseointegration for at least 3 months, then comes the first checkup. At that time, proper implant heads are placed and a previously made temporary denture is fixed to them.

\subsection{Advantages}

One procedure is enough for both osteoplasty, implantation and denture fixation, which means that everything heals at the same time. The patient has a limited chewing ability and gets the final denture after 6 months. During the procedure, the surgeon does not have to adjust or bend the titanium frame as usual. It fits perfectly with the jawbone geometry of the patient so that the surgical procedure is much simpler and faster. The time of the whole procedure and the probability of complications both decrease. For the patient, it means a lower number of procedures, less pain and less 
stress. Moreover, aesthetics becomes less expensive compared to other implantation procedures. In the case of extended bone deficiency, the total time of treatment is between 1-2 years with conventional treatments, but this procedure can decrease it to 3-6 months.

\section{Research history: titanium as an implant material and mechanical testing in dental technology}

Nowadays, finite element analysis and simulation have become an essential part of design processes. It is becoming widespread in numerous industrial applications, also in medical dental technology. Several studies have investigated stress distributions and deformations in the oral cavity, teeth and implants. They focused on the effect of different materials and mechanical loads.

Titanium is the most dominant implant material in dental implantology due to its long-term reliability [6]. The first successful titanium implantation with osseointegration in dentistry was documented in 1977, based on Branemark's experiences. Since then, feedback of follow-ups is constantly positive [7].

We should mention that titanium has some documented disadvantages causing problems e.g. titanium intolerance, electric conduction, corrosion properties, metal-ion aggregation at lymph nodes, and its greyish colour visible through gingival tissues. These factors have led researchers to focus on alternative solutions [6]. As a result of this, titanium is coated with a thin oxide layer that determines the surface properties of the implant. Focusing on its chemical composition, it is mostly made up of $\mathrm{TiO}_{2}$, with a non-crystallized structure and a surface roughness of 0.53-0.67 $\mu \mathrm{m}$. Presumably, this titanium-oxide layer is responsible for the outstanding biocompatibility of titanium implants.
Implants bear with low electric conduction, high corrosion resistance and thermodynamic stability. Furthermore, low ion-release can be detected in humid environments [7].

In a study, stress distributions were examined on five-implant mandibular prosthesis models made up of three different materials with 3D finite element analysis. The examined materials were acrylic resin, metal-acryl, and metalceramic. The result of the investigation was that the acrylic resin model showed the highest stress values in the implant. This proves the necessity of metal reinforcement in acrylic resins [8].

Another study, also using finite element analysis, examined low-quality bone tissue and the possibility of inserting implants into it. According to studies, it can be stated that in low-quality bone tissues, stress concentration and deformation are substantially higher. It is also found that the use of bicortical techniques with the insertion of longer implants decreases stress distribution in neighbouring bone tissue, and also decreases the tendency of deformation [9].

A commonly used finite element software for the calculation and analysis of von Mises stresses is ANSYS. Finite element analysis increases the efficiency of healing, as stress concentrations in bone should be avoided in order to sustain stable osseointegration after implant insertion [10].

\section{Numerical analysis of cortically supported individual implants}

\subsection{Advantages of dual-phase cortically supported implants}

In the recent past, modernization efforts focused on single-phase subperiosteal implants.

The healing of our dual-phase, cortically supported individual implant is more practical. The wound is more protected 
from reinfection and contamination of the future. This is because healing takes place under a closed wound. There is no open scar left for the pillars. A sleeve and an abutment were designed for the cortically supported individual implant. These were designed and verified with numerical analysis.

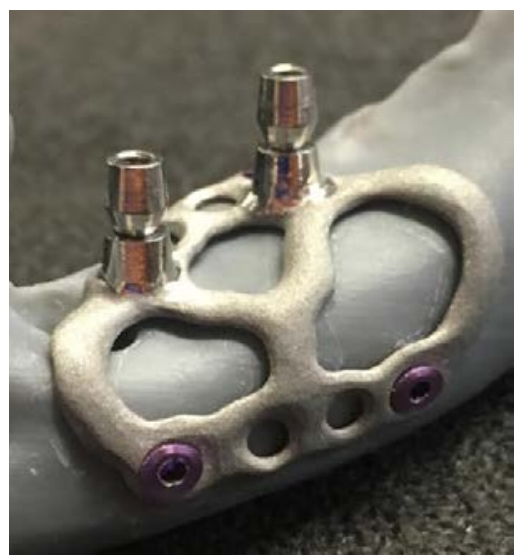

Figure 2. Cortically supported the individual implant

\subsection{Numerical analysis}

\subsubsection{Numerical analysis of dental implants}

The most significant problem of dualphase implant systems is the loosening and/or breaking of the screw. Loosening can be caused by fatigue of the screw due to forces in the oral cavity, or its eccentrical nesting due to implant loading. We examined the problem of loosening using an axially symmetric model. Considering the results, a prestressed screw is used to avoid loosening. The asymmetrical loads lead to such local stress distribution that exceeds the pressing force. Different studies demonstrated that the torque for fixing the implant, suggested by the manufacturer, stays way under the elastic limit. Another probable factor is the behaviour of damping and vibrations that can lead to screw- loosening. However, there were no reports stating that this dynamic behaviour would be a critical factor nearby the jawbone [11].

\subsubsection{The conical fit mechanism in dental implants}

In bolt connection systems, the implantabutment connection is defined by prestressing, which causes a certain torque. The Conical fit mechanism leads to contact pressure that results in friction resistance between the surfaces. Axial forces from chewing can be observed on the surface of the implant. The force arising from chewing helps the connection in the direction of the abutment so that it presses the implant more to the abutment.

Forces from chewing decrease stress intensity in the screw. The conical connection mechanism becomes looser due to tension force and loosening torque. Axial tensile stress enhances the restoration of multi-phase implants. So it can be stated that the solution with conical nesting ensures a secure connection without a third segment (e.g. key, pin and keyway). In the medical field, it is also used in hip replacements. The mechanics of cylindrical close fit is already well-understood. Its relations can be found in different studies, but there is no known solution for conical close fit. The rate of this type of connection depends on bevel angle, connection length, external and internal forces, the degree of connection, material properties, and friction coefficient. Pressure applied on conical surfaces is predicted by functions based on elastic plane stress [12,13].

In practice, the crestal area of the implant is surrounded by cortical bone and the remaining trabecular bone. Clinical studies have shown that bone tissue becomes less dense nearby the implant (osteoporosis). Hereinafter, we assume that bone tissue remains isotropic in material properties, and no osteoporosis occurs [14]. 


\section{Design and implementation}

\subsection{Design of sleeve and abutment for cortically supported dual-phase implants}

Our objective was to design a fitting sleeve and a crown abutment for the cortically supported implant with individual space structure visible in the following figure.

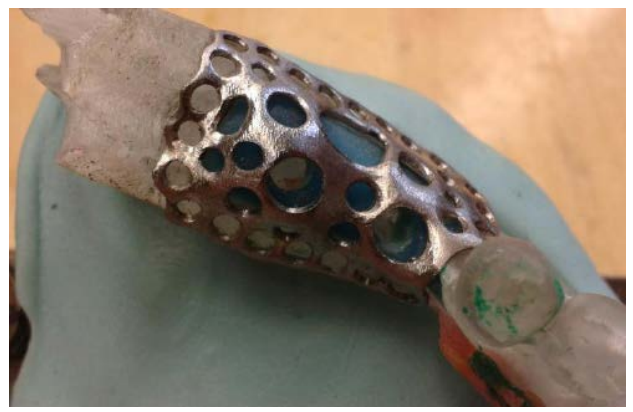

Figure 3. Titanium frame space structure

The bore size of the implant and the size of the sleeve and abutment to be designed were given parameters. The design of all other geometrical dimensions was our task. In this study, we examined the conical connection between the different sleeve and abutment systems. It was preceded by several preliminary experiences. Finite element analysis was adopted for design. Henceforth, we present the research activity carried out.

\subsection{Material properties of the implant}

Commercially pure titanium and titanium alloys are used in medical technology because of their outstanding osseointegration properties, low density, good corrosion resistance, mechanical properties and biocompatibility. A titanium oxide layer is formed on the surface of the implant, which enhances calcium-phosphate production of the organism. This helps speed up the healing process. Titanium and titanium alloys are non-magnetic, so they do not affect medical examinations $[15,16]$.

The following table shows titanium materials commonly used in medical applications. Commercially pure titanium is categorised from Grade I to Grade IV. The higher the grade number the more alloying element it contains. However, up to Grade IV, titanium alloys contain only a small amount of alloying elements. Dental implants are usually made up of $\alpha-\beta$ titanium alloy with $6 \%$ vanadium and $4 \%$ aluminium content. They possess outstanding mechanical load-bearing capacity. The elastic modulus of Grade V alloy is significantly higher than that of human bone. There is no relevant difference between osseointegration properties of $\mathrm{Ti}$ $6 \mathrm{Al}-4 \mathrm{~V}$ alloy and commercially pure titanium $[17,18]$.

Table 1. Classification of titanium and its alloys

\begin{tabular}{|c|c|c|c|c|c|}
\hline Alloy & $\mathrm{E}[\mathrm{GP} \mathrm{a}]$ & $\frac{\text { Elastic limit }}{[\mathrm{MPa}]}$ & $\frac{\text { Tensile }}{\text { strength }}$ & $\varepsilon[\%]$ & Microstucture \\
\hline Cp TiGrade I & 102 & 170 & 240 & 24 & \multirow{4}{*}{ a } \\
\hline Co TiGnde2 & 102 & 275 & 345 & 20 & \\
\hline Cp Ti Grode 3 & 102 & 380 & 450 & 18 & \\
\hline Cp Ti Grade 4 & 104 & 483 & 550 & 15 & \\
\hline Ti-6Al-4V (Grade 5) & 113 & 795 & 860 & 10 & \multirow{2}{*}{$\alpha+\beta$} \\
\hline T1-6Al-4V ELI & 113 & 860 & 930 & 10 & \\
\hline
\end{tabular}

\subsection{Design of sleeve and abutment}

Our goal in the design of sleeve and abutment with individual bevel-angles was to create a bevel-angle that minimizes normal stresses at the conical contact surface with the consideration of bearing surfaces.

In this study, frontal and lateral regions were examined separately. Solo systems were analysed as the outcomes of complex systems would result in a completely different aspect due to force distribution.

Figure 4. shows the three-dimensional and cross-sectional diagram of the sleeve. The design of the outer collar was necessary for its welding to the cortically 
supported individual implant. On that basis, the angle was chosen as $120^{\circ}$. The height of the sleeve was given as $4 \mathrm{~mm}$ as a preliminary design parameter. $4 \mathrm{~mm}$ was chosen by reason of the positioning of bodywork. In dental technology, M2 screws are the most commonly used solutions for implants. Aware of this, the length of the drilled hole was chosen as $3 \mathrm{~mm}$.

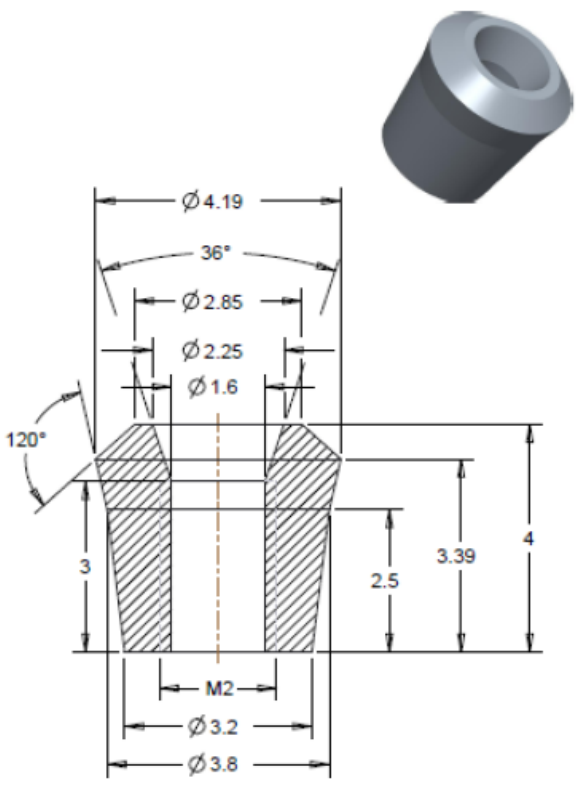

Figure 4. Cross-sectional and $3 D$ diagram of the sleeve

Bevel-angle was determined by finite element analysis. As a result of several trials, which are explained in the next chapter, the final bevel-angle was defined as 36 degrees. The design of the abutment began after finishing the sleeve-design, which can be seen in Figure 5. The height of the abutment and the head diameter were preliminary design parameters as the geometry of bodywork and the height of gum tissue was given. Thus, all other geometrical sizes had to be defined according to these. The design of the abutment can be seen in the following figure.

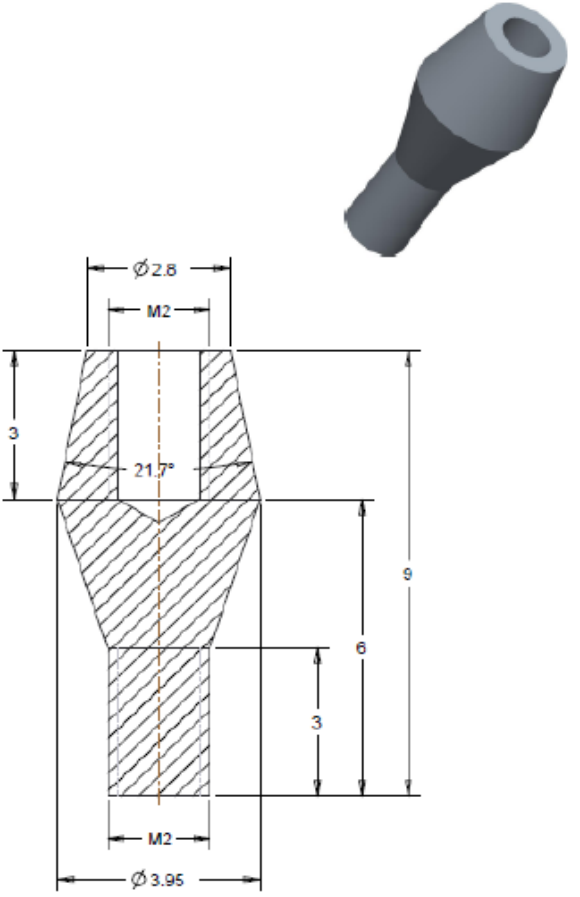

Figure 5. Cross-sectional and $3 D$ diagram of the abutment

\subsection{Numerical analysis}

The results of the numerical analysis can be seen in the following chapter. Examinations were carried out at different bevel-angles between $28^{\circ}$ and $44^{\circ}$, from which significant stress intensities can be visualized. Figure 6. shows stress intensities in the frontal region. In this case, almost vertical forces were calculated. The increase of bevel-angle leads to a marked increase in stress intensity of the system.

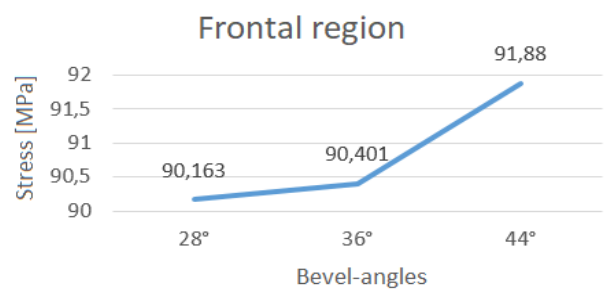

Figure 6. Stress intensities in the frontal region 
The next figure presents stress intensities in the lateral region. It is apparent that the value of stress distribution of the structure decreases with the increase of bevel-angle.

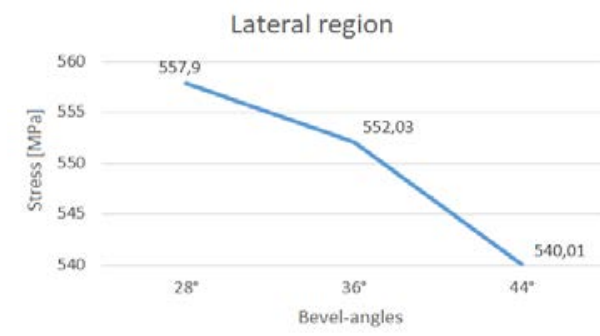

Figure 7. Stress intensities in the lateral region

A solution with a bevel-angle of 36 degrees was chosen according to our analysis. The size of contact surfaces had to be considered as only bevel-angles were altered. Other external geometrical dimensions were not changed. The tapering contact surface can cause mechanical problems that could lead to fracture and failure. As mentioned before, the force is almost perpendicular to the structure in the frontal region. Mechanical stress caused by the vertical force is concentrated to the upper part of the abutment. Thus, the highest stress intensity can be measured in this area.

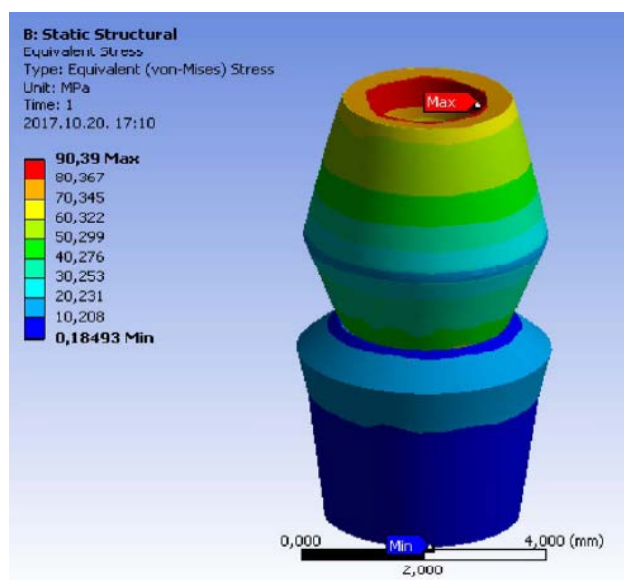

Figure 8. Stress intensities in the frontal region
Mechanical stresses in the lateral region are asymmetrically oriented. The incline angle was chosen as $20^{\circ}$ based on literature studies. The maximum value of stress is measured at the conical connection, which can be observed in the following stress distribution figure.

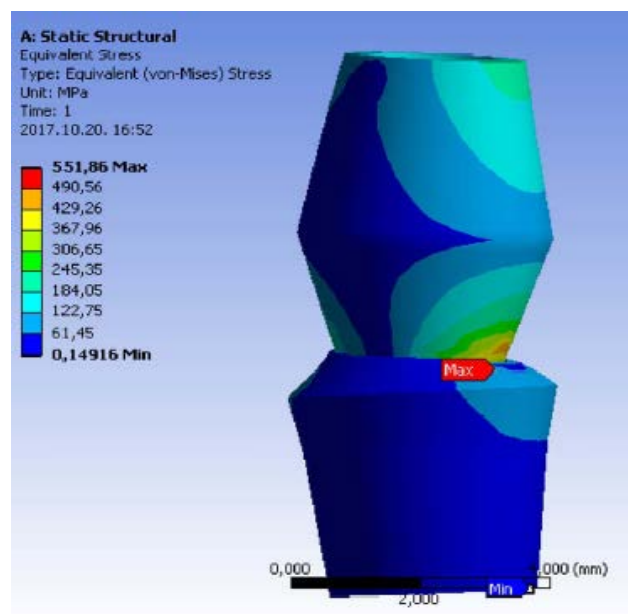

Figure 9. Stress intensities in the lateral region

\section{Conclusions}

This study focuses on the reconsideration and modernization of an old procedure with the use of previously obtained knowledge, and not on the development of a completely new technology. Changes in its original application eliminate the disadvantages of subperiosteal implants but retain their advantages.

The use of new digital techniques (3DCBCT, 3D printing, design software) offers a more precise, more stable, and bettersecured cortically supported individual implant than its original counterpart. Due to the good osseointegration properties of titanium, the new bone tissue is formed in place of the replaceable bone. Moreover, the structural titanium frame will integrate biologically into bone tissue with its total surface - due to bone ingrowth - after 
primer stability is provided by fixing screws.

This surface can be ten times larger than that of conventional implants. Moreover, the implant does not anchor to the weaker trabecular structure of the bone, but to the harder cortical part. It is best applicable for patients who need it most: where other procedures are often not suitable for them.

\section{References}

[1] Dahl G.: Om mojligheten for implantation $i$ kaken av metallskelett som bas eller retention for fasta eller avtagbara proteser. Tidskrift 51 (1943) 440-449.

[2] Vajdovich I.: Dentális implantológia gyakorló fogorvosok részére. Semmelweis Press. Budapest, 2008.

[3] Lin G.-H., Chan H.-L., Wang H.-L.: The significance of keratinized mucosa on implant health: a systematic rewiev. Journal of Periodontol 84/12. (2013) 1755-1767.

https:// doi.org/10.1902/jop.2013.120688

[4] Chang Y. M., Chan S. P., Shen Y. F., Wei F. C.: Soft tissue management using palatal mucosa around endosteal implants in vascularized composite grafts in the mandible. International Journal of Oral \& Maxillofacial Surgery 28/5. (1999) 341-343. https://doi.org/10.1016/S0901-5027(99)80078-2

[5] Bodard A.-G., Salino S., Bémer J., Renaud L., Breton P.: Dental implant placement after mandibular reconstruction by microvascular free fibula flap: current knowledge and remaining questions. Oral Oncology 47/12. (2011) 1099-1104.

https://doi.org/10.1016/j.oraloncology.2011.0 7.016

[6] Kim H.-K., Woo K. M., Shon W.-J., Ahn J.S., Cha S., Park Y.-S.: Comparison of periimplant bone formation around injectionmoulded and machined surface zirconia implants in rabbit tibiae. Dental Materials Journal 34/4. (2015) 508-515.

https://doi.org/10.4012/dmj.2015-024

[7] Sul Y.-T., Johansson C. B., Petronis S., Krozer A., Jeong Y., Wennerberg A., Albrektsson T.: Characteristics of the surface oxides on turned and electrochemically oxidized pure titanium implants up to dielectric breakdown: the oxide thickness, micropore configurations, surface roughness, crystal structure and chemical composition. Biomaterials 23/2. (2002) 491-501.

https://doi.org/10.1016/S0142-9612(01)00131-4

[8] Coelho L. F. B., Brollo J. R., Sartori E. A., Mariano L. O. H., Geremia T., Barcellos L., Corso L. L., Shinkai R. S. A., Grossi M. L.: Stress Distribution Study Using the Finite Element Method in Three Different ImplantSupported Fixed Complete-Arch Mandibular Prostheses. The International Journal of Prosthodontics, 29/3. (2016) 299-302. https://doi.org/10.11607/ijp.4427

[9] Verri F. R., jr. Santiago J., Almeida D. A. de F., Verri A. C. G., Batista V. E. de S., Lemos C. A. A., Noritomi P., Pellizzer E. P.: Threedimensional Finite Element Analysis of Anterior Single Implant-Supported Prostheses with Different Bone Anchorages. The Scientific World Journal, 2015. https://doi.org/10.1155/2015/321528

[10] Markose J., Suresh S., Shruthi E., Rekha K., Vipin J., Supriya M.: Comparison of Platform Switched and Sloping Shoulder Implants on Stress Reduction in various Bone Densities: Finite Element Analysis. The Journal of Contemporary Dental Dental Practice, 18/6. (2017) 510-515.

https://www.ncbi.nlm.nih.gov/pubmed/28621 284

[11] Geng J., Tan K. B. C., Liu G.: Application of finite element analysis in implant dentistry: a review of the literature. The Journal of Prosthetic Dentistry 85/6. (2001) 585-598. https://doi.org/10.1067/mpr.2001.115251

[12] Brunski, J. B.: In vivo bone response to biomechanical loading at the bone/dentalimplant interface. Advanced Dental Research 13/1. (1999) 99-119.

https://doi.org/10.1177/08959374990130012 301

[13] Sutter, F., Weber, H.P., Sorensen, J., Belser, U.: The new restorative concept of the ITI dental implant system: design and engineering. International Journal of Periodontics and Restorative Dentistry 13/5. (1993) 409-431.

[14] Dincer B., Sinan M.: Mechanics of the tapered interference fit in dental implants. Journal of Biomechanics 36/11. (2003) 1649-1658. https://doi.org/10.1016/S00219290(03)00177-5 
[15] Handbook of Materials for Medical Devices (ed.: Davis J. R.). ASM International. Materials Park, USA, 2003.

[16] Orális implantológia. (ed.: Divinyi T.) Semmelweis Kiadó, Budapest, 2007.

[17] Bhola R., Bhola S. M., Mishra B., Olson D. L.: Corrosion in Titanium Dental Implants/
Prostheses. - A review. Trends in Biomaterials and Artificial Organs, 25/1. (2011) 34-46. http://medind.nic.in/taa/t11/i1sji/taat11i1sjip3 4.pdf

[18] Biomaterials: Principles and Applications. (eds.: Park J. B., Bronzino J. D) CRC Press, Boca Raton, 2003. 